ISSN 1112-9867

Available online at

http://www.jfas.info

\title{
QUALITY OF WATERS OF AQUIFER WEBS OF BISKRA REGION
}

\author{
F. Bouchemal, N. Bouchahm and S. Achour
}

Laboratoire de Recherche en Hydraulique Souterraine et de Surface-LARHYSS

Faculté des Sciences et des Sciences de l'Ingénieur

Université de Biskra, BP 145 RP, 07000, Biskra, Algérie

Received: 08 February 2011 / Accepted: 01 June 2010 / Published online: 30 June 2011

\begin{abstract}
Controlling the quality of water distributed together with sound resource management is a factor of economic and social development. Also, the chemistry and knowledge of geological and hydrogeological aquifer, the object of this work, we identify the water quality examined through physical-chemical parameters. The study of these parameters more precisely the region of Biskra reveals a generally high mineralized whose origin is essentially the geological nature of enclosing land. However, the waters of the continental interlayer (Albian) are the chemical profile weakest; however, its high temperature makes it difficult to use both to supply drinking water as well as for irrigation. After synthesis of the results for different sheets (groundwater, Miopliocene, Eocene, Albian), the limestone is most interesting at least for drinking water, part of its rate of mineralization (medium), the acceptable temperature $\left(24^{\circ} \mathrm{C}\right)$ and its relatively low salinity (1 to $3 \mathrm{~g} / \mathrm{l}$ ). However, these waters are not used directly for human consumption. They requires further treatment.
\end{abstract}

Keywords: Aquifer, Biskra, mineralization, chemical profile, quality.

\section{INTRODUCTION}

Dans les pays en voie de développement à climat aride, le rôle des eaux souterraines est d'autant plus important qu'elles constituent souvent la seule source d'approvisionnement en eau potable et sont donc vitales pour le développement de ces pays (TRAVI, 1993).

Author Correspondence, e-mail: bouchemal_hydro@yahoo.fr

ICID: 1020773 
Pour le Sahara Septentrional Algérien, l'essentiel des ressources est constitué par les eaux souterraines. Ces dernières, contenues dans les formations continentales du continental intercalaire (C.I) et du complexe terminal (C.T) constituent l'un des plus vastes réservoirs hydrauliques du monde (BEL et CUCHE, 1970 ; CDTN, 1992), dont les potentialités mobilisables sont estimés à 5 milliards de mètres cube d'eau (ANRH, 1986).

Les conditions lithologiques, hydrodynamiques et hydro-chimiques sont variées, notamment dans la partie Est de la région, et confèrent de ce fait un intérêt particulier à ces nappes (ERESS, 1972). Par ailleurs, diverses études entreprises depuis une trentaine d'années, ont montré que les eaux de cette région sont caractérisées par une minéralisation totale excessive, le plus souvent associées à une dureté élevée et des concentrations élevées en fluorures (ACHOUR, 1990 ; AZOUT et ABRAHAM, 1978 ; PINET et al ., 1961).

La wilaya de Biskra présente des ressources en eau souterraines très importantes, représentées par les nappes des deux systèmes aquifères du Sahara septentrional, le complexe terminal et le continental intercalaire. (ERESS, 1972).

L'exploitation des eaux souterraines est la principale ressource pour l'alimentation en eau potable pour les habitants de la région de Biskra. En fait l'exploitation de deux barrages sur la région ne se fait que pour l'irrigation des cultures.

Les caractéristiques chimiques de l'eau ont un impact déterminant non seulement au niveau da la santé publique et de la potabilité de l'eau mais encore au niveau de certains secteurs, et en particulier l'agriculture qui est de loin celui qui consomme le plus d'eau au Sahara (ACHOUR, 1990)

Le principal objectif de cette étude est de donner un aperçu sur la qualité physico chimique des eaux du Biskra. Nous nous sommes particulièrement intéressé aux eaux du complexe terminal.

\section{MATERIELS ET METHODES}

\subsection{Echantillonnage}

Les prélèvements d'échantillons sont effectués dans des flacons en polyéthylène de 1000 $\mathrm{ml}$ de capacité.

Le flacon est rincé au préalable avec l'eau à prélever. Lorsqu'il s'agit d'un forage d'irrigation, l'échantillon est pris directement à la tête de celui-ci. Dans le cas d'un 
château d'eau, le prélèvement est effectué après suspension du processus de javellisation et vidange complète de la conduite afin que l'échantillon soit réellement représentatif de l'eau du forage (BOUCHAHM, 1999).

\subsection{Méthodes analytiques}

Nous avons effectué les analyses physico-chimiques des échantillons d'eaux au sein du laboratoire Qualité et Traitement des Eaux Souterraines et de Surface «LARHYSS ». Les procédures de dosage sont déduites des méthodes d'analyse standard.

La conductivité est déterminée grâce à un conductimètre électrique

(WEILHEIM LF 90) qui donne directement la conductivité de l'échantillon, à la température adoptée $\left(25^{\circ}\right)$ en $\mathrm{ms} / \mathrm{cm}$ ou en $\mu \mathrm{s} / \mathrm{cm}$.

La dureté totale des échantillons est déterminée par titrage avec l'acide éthylène diamine-tétracétique (EDTA) qui complexe quantitativement de nombreux métaux, dont le $\mathrm{Ca}^{2+}$ et $\mathrm{Mg}^{2+}$; ceci dans un milieu tamponné entre 9,5 et 10 en présence du noir d'ériochrome T comme indicateur (RODIER, 1996).

La mesure de l'alcalinité est basée sur la neutralisation d'un certain volume de l'échantillon par l'acide sulfurique (N/50), en présence de phénophtaléine $(0,5 \%)$ pour la détermination du TA, et du méthylorange $(0,5 \%)$ pour la détermination du TAC (RODIER, 1984).

Les chlorures sont déterminés par la méthode de Mohr (RODIER, 1996).

Les ions chlorure réagissent quantitativement avec le nitrate d'argent, avec formation de chlorure d'argent, sel très peu soluble. On utilise le chromate de potassium $\mathrm{K}_{2} \mathrm{CrO}_{4}$ comme indicateur.

Les sulfates sont dosés par colorimétrie en utilisant un photomètre (palintest 5000) réglé à une longueur d'onde de $520 \mathrm{~nm}$ en utilisant un réactif spécifique.

Le sodium et le potassium sont dosés par photométrie à émission de flamme sur appareil JENWAY PFP7.

Le fluor est dosé par la méthode potentiométrique. Le dosage s'effectue grâce à une électrode spécifique aux ions fluorures (ELIT8221F-) et une électrode de référence au chlorure d'argent (ELIT001AgCl 56113). L'établissement de courbes d'étalonnage reliant les concentrations de l'ion fluorure au paramètre mesuré a été nécessaire. 


\section{DISCUSSION ET INTERPRETATION DES RESULTATS}

\section{3-1- Le pH}

\section{a) La Nappe phréatique}

Sur l'ensemble des forages échantillonnés la nappe phréatique montrent des $\mathrm{pH}$ allant de à 7,14 et 8,3 .

\section{b) Nappe du Miopliocène}

Les valeurs du $\mathrm{pH}$, mesurées pour des échantillons eaux des forages captant le niveau Miopliocène, montrent que le $\mathrm{pH}$ de ces eaux varie entre 7,26 et 8,12.

\section{c) Nappe de l'Eocène}

Pour cette nappe captée à Tolga et Doucen, la mesure du pH des eaux des forages de cette nappe donne des valeurs variant entre 7,00 et 8,3.

\section{d) Nappe de l'Albien}

Les forages dans la nappe de l'Albien pour la région ne sont pas encore nombreux, pour les quelques forages dont on a pu avoir des échantillons, les valeurs du pH mesuré varient entre 7,7 et 8,3 .

$\mathrm{Au}$ cours de tous ces résultats, il apparaît que le $\mathrm{pH}$ de toutes les eaux est voisin de la neutralité avec un caractère plus ou moins alcalin. La plupart des eaux présentent aussi un caractère alcalin bicarbonaté du fait que le pH est souvent inférieur à 8,3.

\section{3-2- La conductivité}

\section{a) Nappe phréatique}

Pour cette nappe phréatique, la conductivité en $(\mathrm{ms} / \mathrm{cm})$, varie entre une valeur minimale de 2,86 (forage Doucen IRR) et une valeur maximale de 7,63 (forage $\mathrm{N}^{\circ} 111$ ).

\section{b) Nappe du Miopliocène}

Les valeurs mesurées de la conductivité, pour les échantillons de la nappe du Miopliocène, varient entre une valeur minimale de 1,79ms/cm (Forage $\mathrm{N}^{\circ}$ 370/321) et atteignent une valeur maximale de $5,53 \mathrm{~ms} / \mathrm{cm}$ (Forage $\mathrm{N}^{\circ} 156$ ).

\section{c) Nappe de L'Eocène}

La conductivité, pour la nappe des calcaires, varie entre une valeur minimale de $\mathrm{ms} / \mathrm{cm}$ (Forage $\mathrm{N}^{\circ} 150$ ) et une valeur maximale de 5,8 ms/cm (Forage $\mathrm{N}^{\circ} 153$ ).

\section{d) Nappe de l'Albien}


Pour les eaux de l'Albien, la conductivité mesurée varie entre 3,0 ms/cm (forage $\mathrm{N}^{\circ}$ 94) et $4,5 \mathrm{~ms} / \mathrm{cm}\left(\operatorname{SCE~N}{ }^{\circ} 6\right)$.

Toutes les valeurs mesurées de la conductivité indiquent une minéralisation élevée car elles sont toutes globalement supérieures à $1000 \mu \mathrm{s} / \mathrm{cm}$. De plus, elles correspondent à des valeurs de minéralisation totale dépassant les normes de l'O.M.S. (soit $1500 \mathrm{mg} / \mathrm{l}$ ).

\section{3-3- Le titre hydrotimétrique}

Les valeurs de la dureté observées, pour tous les échantillons des nappes de la région d'étude, sont classées en valeurs minimales, maximales et moyennes dans le tableau suivant:

Tableau 1. Dureté des eaux de la région d'étude

\begin{tabular}{|l|c|c|c|}
\hline \multicolumn{1}{|c|}{ Nappe } & $\begin{array}{c}\text { Valeur } \\
\text { Min } \\
\left({ }^{\circ} \mathbf{F}\right)\end{array}$ & $\begin{array}{c}\text { Valeur } \\
\text { Max } \\
\left({ }^{\circ} \mathbf{F}\right)\end{array}$ & $\begin{array}{c}\text { Valeur } \\
\text { Moy } \\
\left({ }^{\circ} \mathbf{F}\right)\end{array}$ \\
\hline -Phréatique & 52 & 259 & 115,41 \\
-Miopliocène & 74 & 386 & 181,2841 \\
-Eocène & 24,5 & 82,38 & 46,35 \\
-Albien & 33,36 & 191 & 68,93 \\
& & & \\
\hline
\end{tabular}

D'après les normes de potabilité des eaux établies par l'O.M.S, on remarque que pour les échantillons observées, le titre Hydrotimétrique moyen dépasse $54{ }^{\circ} \mathrm{F}$; ce qui montre que les eaux de cette région sont très dures, sauf ce qui concerne la nappe de l'éocène dont les eaux sont moins dure $\left(46,35^{\circ} \mathrm{F}\right)$.

\section{3-4- Faciès chimique}

Les nombreuses données physico-chimiques, utiles à la caractérisation d'une source d'eau minérale, induisent l'utilisation de représentations graphiques. L'objectif principal d'une représentation graphique est de permettre une approche rapide des résultats analytiques en vue de caractériser facilement une eau, de suivre son évolution ou de la comparer à d'autres eaux minérales de compositions physico-chimiques 
voisines ou de proximité géographique. Les deux graphiques, Schoeller et Piper sont les représentations des concentrations en ions majeurs les plus courantes.

Les points essentiels à retenir sont précisés ci-après :

$>$ Le diagramme de Schoeller est bien adapté à la comparaison d'échantillons de faciès identique mais différents par leur degré de dilution, avec cependant le risque d'interprétation abusive de la conformité de 2 échantillons du fait des reports graphiques sur échelle logarithmique.

Le diagramme de Piper est bien adapté pour l'étude comparative d'un nombre important d'échantillons, présente le risque d'erreur dans la comparaison d'échantillon du fait d'une représentation en \% des ions.

\section{a) Nappe phréatique}

Représentées sur les deux diagrammes de Piper et de Schoeller (cf. fig1), les eaux de la nappe phréatique sont de faciès :

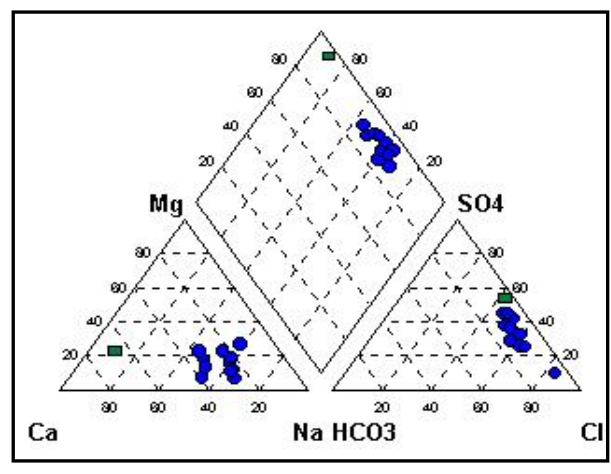

Fig.1a. Diagramme de Piper

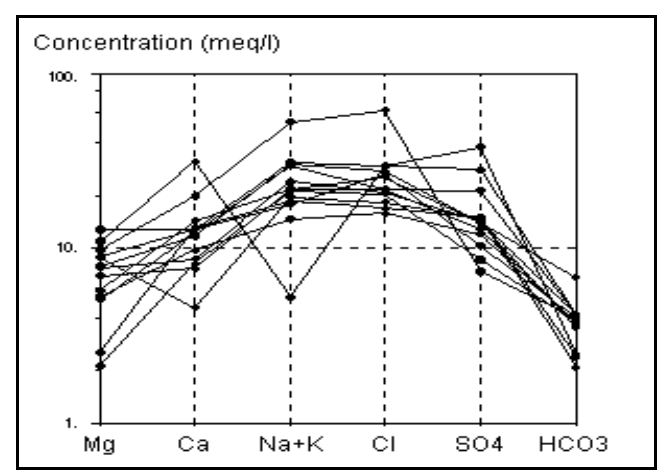

Fig.1b. Diagramme de Schoeller

Les eaux de cette nappe présente un faciès chloruré sodique et potassique avec la tendance de quelques forages vers le pôle sulfaté calcique et magnésienne. Un seul forage présente le faciès hyperchloruré calcique sulfaté.

Les ions dominants sont les chlorures pour les anions et le sodium et potassium pour les cations.

Si on le remarque bien, les eaux de cette nappe phréatique se chargent de plus en plus de matières dissoutes. La distribution montre une nette dominance des chlorures et des sulfates sur le calcium et le magnésium. 
Selon les formules caractéristiques, les ions s'ordonnent de la manière suivante :

- Pour le premier faciès : chloruré sodique et potassique

$$
\mathrm{Cl}^{-}>\mathrm{SO}_{4}^{--}>\mathrm{HCO}_{3}^{-} \text {et } \mathrm{Na}^{+}>\mathrm{Ca}^{++}>\mathrm{Mg}^{++}
$$

- Pour le deuxième faciès : chloruré et sulfaté calcique et magnésienne

$$
\mathrm{SO}_{4}^{--}>\mathrm{Cl}^{-}>\mathrm{HCO}_{3}^{-} \text {et } \mathrm{Ca}^{++}>\mathrm{Mg}^{++}>\mathrm{Na}^{+}
$$

\section{b) Nappe du Miopliocène :}

Les eaux de la nappe du Miopliocène présentent (Fig.2) :
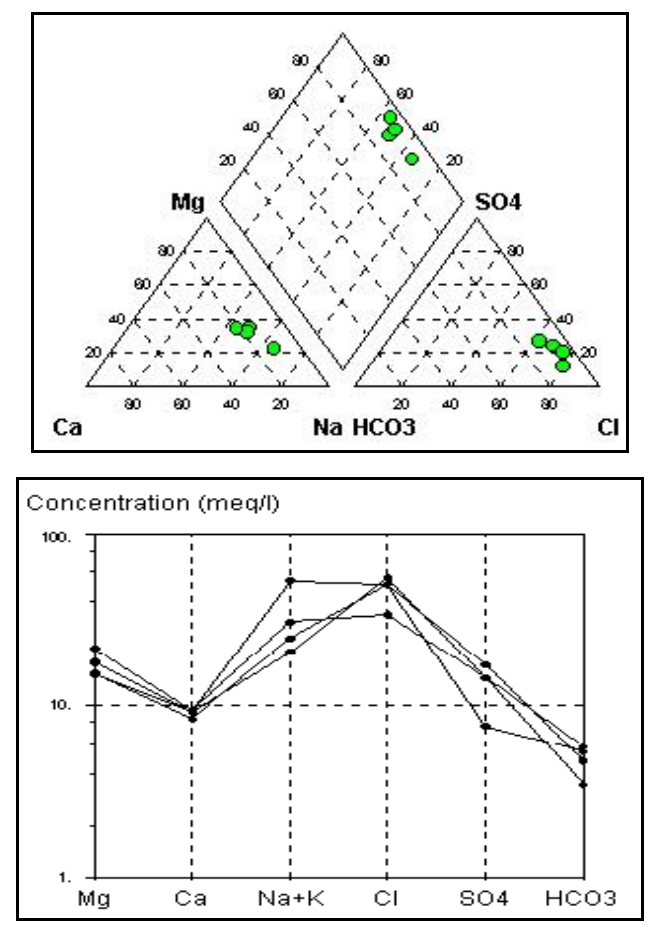

Fig.2a. Diagramme de Piper

Fig.2b. Diagramme de Schoeller

Deux faciès, le premier chloruré sodique et potassique et le deuxième chloruré et sulfaté cacique et magnésien.

- Les cations dominants sont le sodium et le potassium pour le premier faciès, avec tendance du calcium et magnésium pour le deuxième pôle.

- Les anions dominants sont les chlorures $\mathrm{Cl}^{-}>\mathbf{S O}_{4}^{--}>\mathbf{H C O}_{3}^{-}$,

\section{c) Nappe de l'Eocène}

Présentées sur le diagramme de Piper (Fig. 3) les eaux de cette nappe sont de type : sulfaté calcique et magnésienne.

Un ou deux forages présentent le faciès chloruré sodique et potassique. 
Les ions dominants sont les sulfates pour les anions et les calcium et magnésium pour les cations.
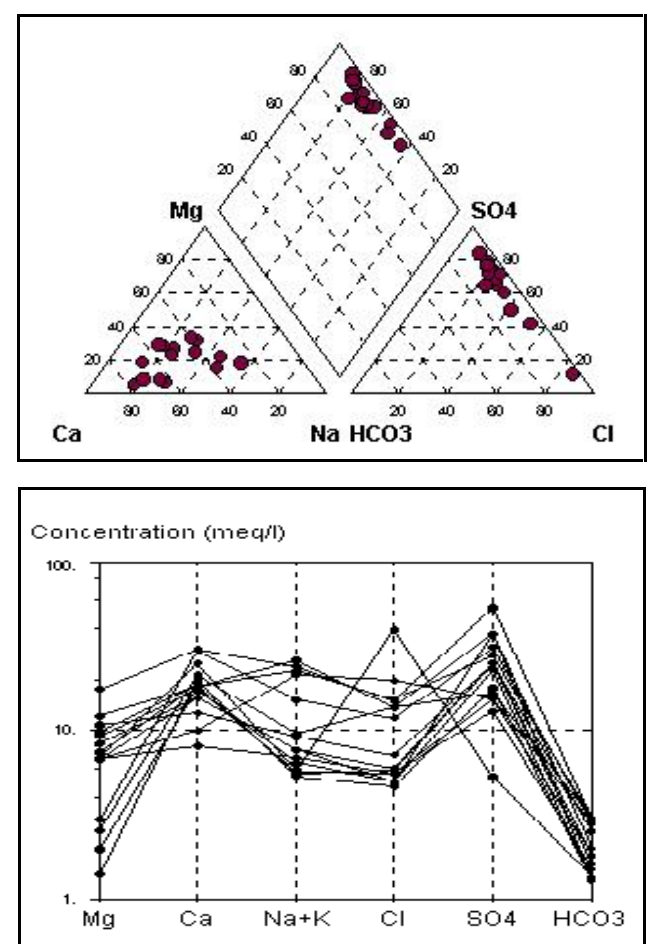

Fig.3a. Diagramme de piper

Fig.3b. Diagramme de Schoeller

d) Nappe de l'Albien

Les eaux de cette nappe sont de type sulfaté calcique et magnésien (cf. Fig 4) avec des tendances au pôle chloruré sodique et potassique. 

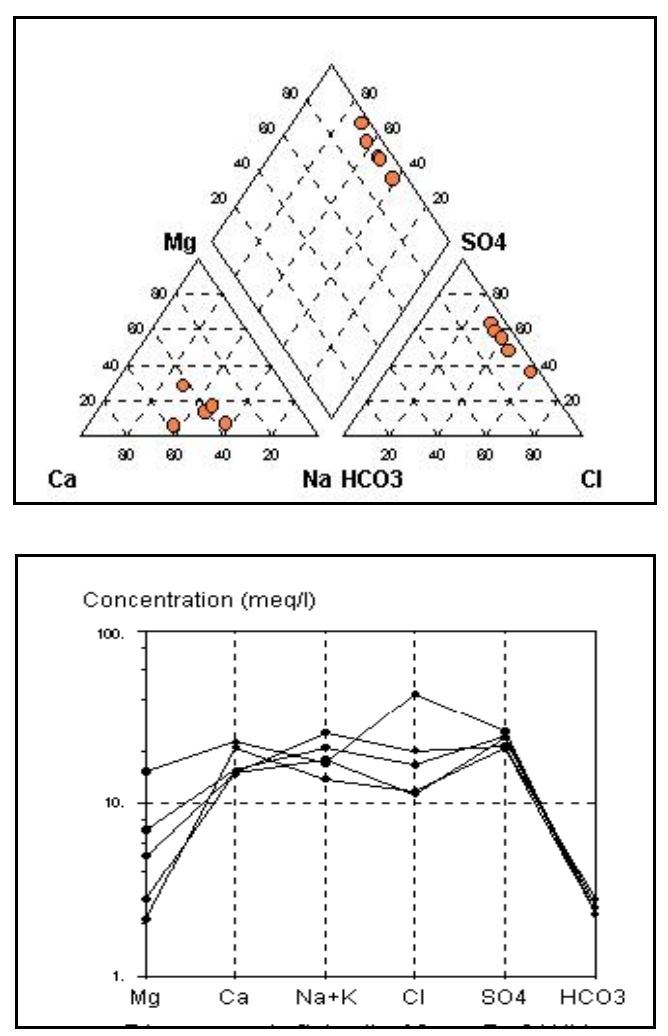

Fig.4a. Diagramme de Piper

Fig.4b. Diagramme de Schoeller

Ainsi, à quelques exceptions prés, nous pouvons considérer que toutes les eaux échantillonnées sont de type sulfaté- chloruré avec pour cations dominants le calcium et le magnésium avec toujours la tendance vers le pôle chloruré ce qui explique un enrichissement en sels par dissolution.

\section{3-5-Teneur en fluor}

Le fluor est présent dans toutes les eaux des nappes de la région et varie d'un aquifère à l'autre. Dans la nappe phréatique il vari entre une concentration minimale de 1,07 mg/l et une valeur maximale de 3,67 mg/l. pour la nappe sous- jacente, le Miopliocène sa teneur varie entre $0,58 \mathrm{mg} / \mathrm{l}$ et $3,83 \mathrm{mg} / \mathrm{l}$.

La concentration la mois élevée se rencontre dans la nappe de l'albien est de 0,65mg/l.

On remarque ici que les concentrations en fluor des eaux souterraines de cette région dépassent les normes de potabilité qui sont de $1 \mathrm{mg} / \mathrm{l}$ de fluor dans l'eau de boisson.

\section{CONCLUSION}

L'objectif de notre travail était d'étudier les caractéristiques physico - chimiques relatives à la qualité des eaux de la région de Biskra, pour différents niveaux aquifères. 
Nous avons utilisé une variété de méthodes analytiques dans le dosage des éléments minéraux.

Il nous a été possible d'étudier l'évolution des principaux paramètres de qualité physico-chimiques tant du point de vue géographique et géologique.

En particulier, les conductivités relevées correspondent à des minéralisations totales importantes diminuant avec la profondeur en passant de la nappe phréatique vers la nappe albienne.

Les ions dominant sont le plus souvent les chlorures et les sulfates ainsi que le calcium et le magnésium.

Quant au fluor, les teneurs les moins élevées se trouvent dans la nappe de l'Albien. Concernant les autres nappes, les teneurs dépassent dans tous les cas la norme de potabilité.

D'une façon générale, les caractéristiques minérales des eaux de la région d'étude sont apparues globalement médiocres avec des valeurs non-conformes aux normes de potabilité, notamment en ce qui concerne les nappes les plus exploitées (Nappes du complexe terminal).

\section{REFERENCES BIBLIOGRAPHIQUES}

[1] ACHOUR. S, (1990): la qualité des eaux du Sahara Septentrional en algérie. Etude de l'excès en fluor, Tribune de l'eau, Cebedeau, $\mathrm{n}^{\circ} 6,42$ (542), 53-57.

[2] A.N.R.H (1986): Ressources en eau et en sols de l'Algérie, rapport.

[3] AZOUT, B., ABRAHAM, J. (1978): Existence et causes des fluoroses humaines dans la région d'El Oued, Annales de l'I.N.A.,volume VIII, 3, 5-12 Alger

[4] BEL, F\& CUCHE, D., (1970) : Etude des nappes du complexe Terminal du bas Sahara.

[5] Données géologiques et hydrogéologiques pour la construction du modèle mathématiques DHW.Ouargla.

[6] BOUCHAHM .N, (1999) : Etude de la répartition spatiale des teneurs en fluorures des eaux du Sahara Septentrional, thèse de Magistère en hydraulique, université de Biskra.

[7] CDTN (rapport) (1992) : Etude hydrochimique et isotopique des eaux souterraines de la cuvette de Ouargla. Centre de développement des techniques nucléaires. $1^{\mathrm{er}}$ Rapport : $1^{\text {ère }}$ campagne novembre 1991. $2^{\text {ème }}$ Rapport : $2^{\text {ème }}$ campagne 1992. 
[8] E.R.E.S.S, (1972). Etude des ressources en eau du Sahara septentrional. Par UNESCO. Utilisation des modèles pour l'établissement des programmes d'exploitation. Vol 5. Nappe du continental intercalaire. Vol 2. Nappe du complexe terminal. Vol 3.

[9] PINET,F., BARRIERE, J., BOUCHE, B (1961) : Endemic fluorosis of aqueous origin in Souf. Darmous and fluorosis osteoporoses.Areport of 51 cases of condensing osteoses, Algérie Med, 65.

[10] RODIER J, (1984). L'analyse de l'eau, eaux naturelles, eaux résiduaires, eau de mer, $7^{\text {ème }}$ édition, Ed. Dunod, Paris.

[11] RODIER J, (1996). L'analyse de l'eau, eaux naturelles, eaux résiduaires, eau de mer, $8^{\text {ème }}$ édition, Ed. Dunod, Paris.TRAVI, Y., (1993). hydrogéologie et hydrochimie des aquifères du Sénégal, hydrochimie du fluor dans les eaux Souterraines,), Sciences géologiques, Louis Pasteur et CNRS. 


\section{QUALITE DES EAUX DES NAPPES AQUIFERES DE LA REGION DE BISKRA}

\section{RESUME}

La maîtrise de la qualité de l'eau distribuée associée à une bonne gestion de la ressource constitue un facteur de développement économique et social.

Par ailleurs, l'hydrochimie et la connaissance des formations géologiques et hydrogéologiques des aquifères, objet de ce travail, nous permettent d'identifier la qualité de l'eau examinée à travers les paramètres physico-chimiques. L'étude de ces paramètres plus précisément pour la région de Biskra révèle en générale une minéralisation élevée dont l'origine est essentiellement la nature géologique des terrains encaissants. Toutefois, les eaux du continental intercalaire (albien) présentent le faciès chimique le plus faible, néanmoins sa température excessive la rend difficilement utilisable aussi bien pour l'A.E.P que pour l'irrigation. Après synthèse des résultats relatifs aux différents nappes (Phréatique, Miopliocène, Eocène, Albien), celle des calcaires reste la plus intéressante du moins pour l'A.E.P, de part son taux de minéralisation (moyen), sa température acceptable $\left(24^{\circ} \mathrm{C}\right)$ et sa salinité relativement peu élevée (1 à $3 \mathrm{~g} / \mathrm{l})$. Cependant, ces eaux ne sont pas utilisables directement pour la consommation humaine. Elles nécessitant un traitement plus poussé.

Mots clés : Aquifère, Biskra, minéralisation, faciès chimique, qualité

\section{How to cite this article}

Bouchemal F, Bouchahm N and Achour S. Quality of waters of aquifer webs of Biskra region. J Fundam Appl Sci. 2011, 3(1), 35-46. 